\title{
'SW', a New Watermelon Cultivar with a Sweet and Sour Flavor
}

\author{
Lei Gao, Shengjie Zhao, Xuqiang Lu, Nan He, and Wenge Liu' \\ Zhengzhou Fruit Research Institute, Chinese Academy of Agricultural \\ Sciences, Zhengzhou, Henan 450009, China
}

Additional index words. watermelon, breeding, soluble sugars, organic acids

Improving living standards have led to increasing interest in the quality of watermelon [Citrullus lanatus (Thunb.) Manf.] fruit among producers and consumers; thus, the cultivation of high-quality watermelon varieties that meet consumer demands is particularly important. Watermelon fruit quality includes commodity quality, organoleptic quality, nutritional quality, and more. Organic acid composition and content are thought to play an important role in the organoleptic quality, nutritional quality, and metabolism of the fruit (Silva et al., 2004; Zampini et al., 2008). Furthermore, organic acids have important nutritional value for the human body; the natural flavor malic acid is conducive to amino acid absorption, whereas citric acid can increase appetite and promote calcium and phosphorus absorption. Different consumers have various preferences for sweet and sour fruit flavor because of dietary changes; when different varieties have a high soluble sugar fruit content, the cultivar with high organic acid content is more popular (Wu et al., 2003). In China, the Cantaloupe Research Center of Xinjiang Academy of Agricultural Sciences has successfully cultivated a series of melon varieties with sweet and sour flavor, which have broad potential applications. The main organic acid of watermelon fruit is malic acid, followed by citric acid (Chisholm and Picha, 1986). However, most cultivated watermelon varieties worldwide are sweet; other varieties with a sweet and sour flavor remain limited in the current market. The aim of this research was to develop a new watermelon cultivar with a sweet and sour flavor, which could fill this market void and satisfy consumer demands for watermelon fruit.

\section{Origin}

' $\mathrm{SW}$ ' was derived from a cross between cultivated watermelon inbred '203Z' and

Received for publication 16 Jan. 2018. Accepted for publication 9 Apr. 2018.

This research was supported by the China Agriculture Research System (CARS-25-03), grants from the Agricultural Science and Technology Innovation Program (CAAS-ASTIP-2018-ZFRI), the National Natural Science Fund of China (31672178 and 31471893), and the Central Public-interest Scientific Institution Basal Research Fund (No. 1610192018109).

${ }^{1}$ Corresponding author. E-mail: 1wgwm@163.com. wild watermelon subspecies 'PI271769'. '203Z' and 'PI271769', used as breeding line and germplasm resources, were conserved at the Zhengzhou Fruit Research Institute, Chinese Academy of Agricultural Sciences. '203Z' is an elite breeding line that has spherical fruit with green rind, dark green pencil stripes at the mature fruit stage, pink red flesh, a high total soluble sugar content, and a low total organic acid content, whereas 'PI271769' is a plant introduction from the U.S. Department of Agriculture germplasm collection and has spherical pale green fruit, a low total soluble sugar content, and a high total organic acid content with white flesh at the mature fruit stage. Development of 'SW' began in the Spring of 2011 at the polyploidy watermelon breeding experimental station in Zhengzhou, Henan Province, and Sanya, Hainan Province. Initially, a cross between '203Z' (used as the female parent) and 'PI271769' (used as the male parent) was produced. Next, the $F_{1}$ plants were backcrossed seven times with '203Z' as the recurrent parent to generate $\mathrm{BC}_{7} \mathrm{~F}_{1}$. Controlled self-pollination was then performed four times to generate $\mathrm{BC}_{7} \mathrm{~F}_{5}$. At every generation, the five fruit with high total soluble sugar content and high total organic acid content were selected and their seeds collected; these were grown in a greenhouse with controlled pollination. Finally, the new stable watermelon cultivar with high total soluble sugar content and high total organic acid content was obtained in the Fall of 2015; this cultivar was named 'SW'.

\section{Plant Characteristics}

About 50 plants each of 'SW', '203Z', and 'Sugar Baby' (used as control material) were evaluated during the Summer and Fall of 2016 in Xinxiang, Henan Province. Standard conventional field cultivation was followed during the growing season in the greenhouse. Seedlings at the third true-leaf stage were transplanted in a plastic greenhouse, and fertilizer, irrigation application, and pest control were implemented following the standard practices. Simultaneously, artificial self-pollination was performed and only one fruit per plant was allowed to develop. Based on observed botanical characteristics, including root, stem, leaf, flower, and fruit, there is no significant phenotypic difference between 'SW' and '203Z'. 'SW' plants produced spherical fruit with green rind and dark green pencil stripes, and pink-red flesh similar to '203Z' (Fig. 1). Flesh sample homogenates from the center of watermelon fruit were used to determine fruit soluble solid content (expressed as a percentage) and $\mathrm{pH}$ with a laboratory refractometer and a $\mathrm{pH}$ meter (Shanghai LICHENKEYI, China), respectively. Once the fruit flesh total soluble sugar content and $\mathrm{pH}$ were established, the pooled juices were collected, rapidly frozen in liquid nitrogen, and immediately stored at $-80{ }^{\circ} \mathrm{C}$ until used to measure soluble sugar (glucose, fructose, and sucrose) and organic acid (malic acid and citric acid) content, as previously described (Bartolozzi et al., 1997). The soluble sugar and organic acid content values of ripe fruits are shown in Fig. 2. In mature '203Z', 'Sugar Baby', and 'SW' watermelon fruit, fructose, sucrose, and glucose were the major soluble sugars, whereas the organic acids included malic and citric acid; these results are consistent with previous reports (Chisholm and Picha, 1986; Zhang and Ge, 2016; Zhu et al., 2017). Fructose, sucrose, and the soluble solid content were lower in 'SW' than in '203Z' and 'Sugar Baby' (Fig. 2A and B); however, there was no significant difference between the watermelons from the same season. By contrast, the citric acid and especially malic acid contents were much higher in ' $\mathrm{SW}$ ' than in '203Z' and 'Sugar Baby' (Fig. 2C) and there was a significant difference between the different varieties from the same season. The soluble sugar and organic acid contents were easily influenced by environmental factors and genetic factors. In the same cultivar, the soluble sugar content was much higher in the summer than in the fall, whereas the organic acid content showed the reverse trend (Fig. 2). Overall, 'SW' has a higher total organic acid content at the mature stage than '203Z'; however, there were no other
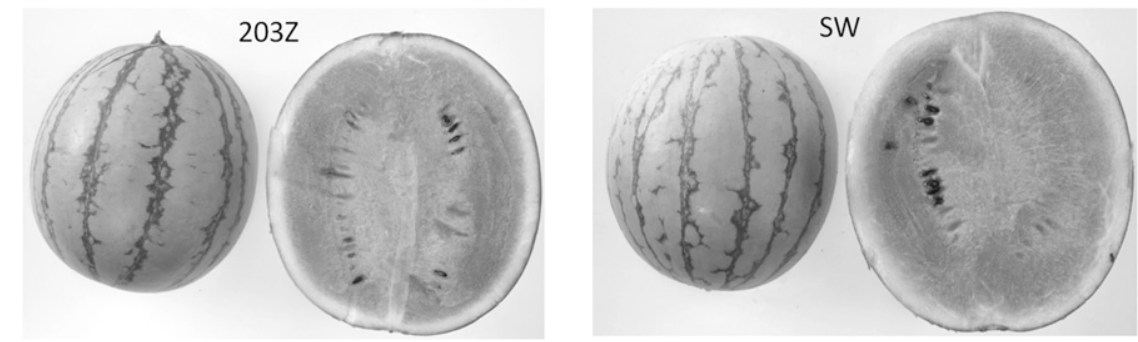

Fig. 1. Ripe '203Z' and 'SW' fruit showing spherical fruit, rind pattern and thickness, and pink-red flesh. 

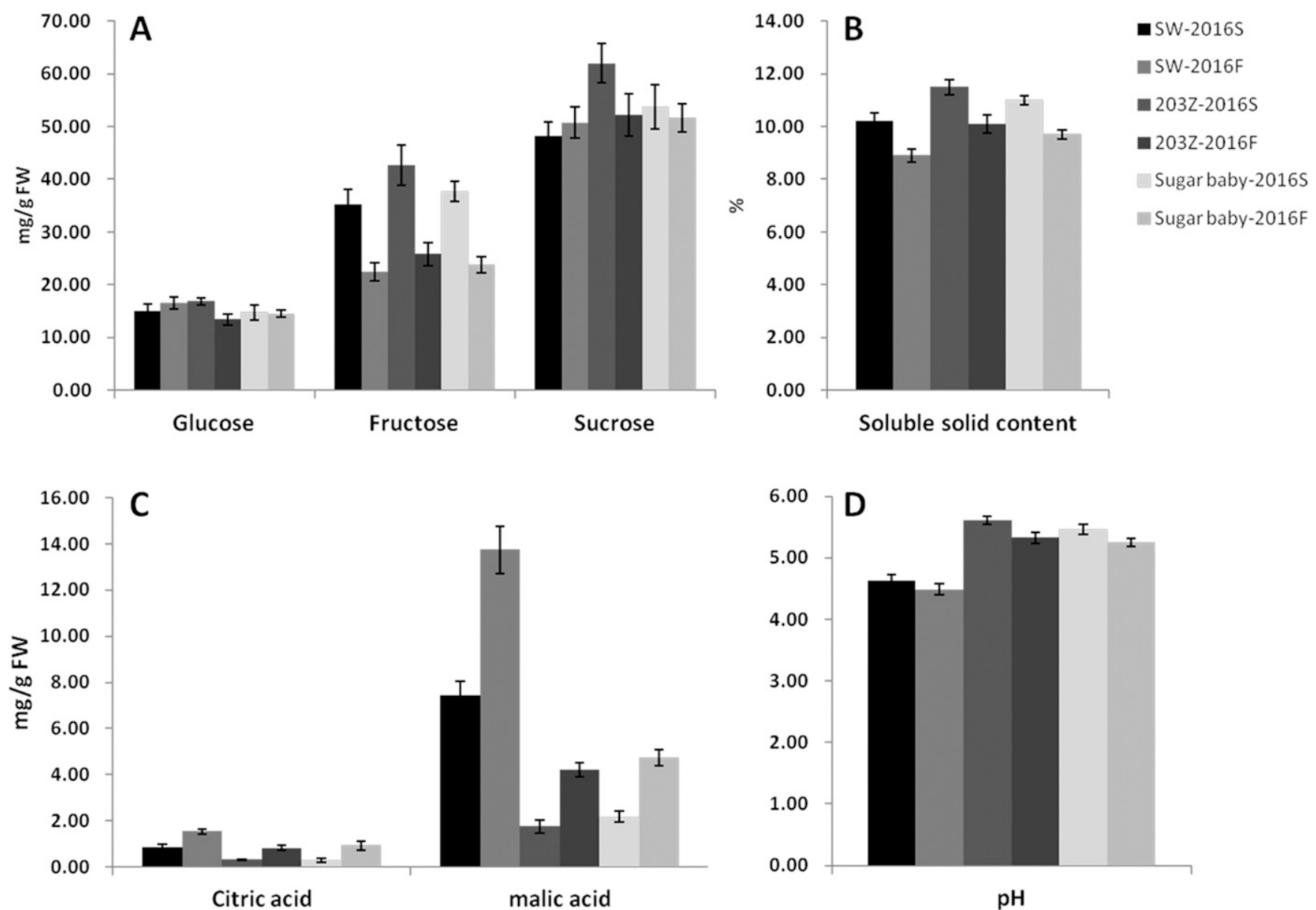

Fig. 2. The content of soluble sugars (glucose, fructose, and sucrose) (A), soluble solid content (B), organic acids content (malic acid and citric acid) $(\mathbf{C})$, and pH (D) in ripe 'SW', '203Z', and 'Sugar Baby' fruit in the Summer and Fall of 2016. The bars represent mean $\pm \operatorname{SE}(n=5)$.

obvious differences among other characteristics of these varieties.

' $\mathrm{SW}$ ' is a special watermelon with high malic acid content that would appeal to consumers interested in sweet and sour flavor. In addition, 'SW' can be used as breeding material for the development of new watermelon varieties with a sweet and sour flavor for specialty markets.

\section{Availability}

Small samples of ' $\mathrm{SW}$ ' seeds are available for distribution to research personnel and watermelon breeders who submit a written request to Dr. Wen-ge Liu, Zhengzhou Fruit Research Institute, Chinese Academy of Agricultural Sciences, Zhengzhou, Henan Province, China (e-mail: lwgwm@163. com). 'SW' seeds will also be submitted to the National Watermelon and Melon Gene
Bank where they will be available for research purposes including the development and commercialization of new watermelon cultivars. It is requested that appropriate recognition of the source be given when this germplasm contributes to the research or development of a new breeding line or cultivar.

\section{Literature Cited}

Bartolozzi, F., G. Bertazza, D. Bassi, and G. Cristoferi. 1997. Simultaneous determination of soluble sugars and organic acids as their trimethylsilyl derivatives in apricot fruits by gas-liquid chromatography. J. Chromatography 758:99-107.

Chisholm, D.N. and D.H. Picha. 1986. Distribution of sugars and organic acids within ripe watermelon fruit. HortScience 21:501-503.

Silva, B.M., P.B. Andrade, A.C. Gonçalves, R.M. Seabra, M.B. Oliveira, and M.A. Ferreira 2004. Influence of jam processing upon the contents of phenolics, organic acids and free amino acids in quince fruit (Cydonia oblonga Miller). Eur. Food Res. Technol. 218:385-389.

$\mathrm{Wu}$, B., B. Quilot, J. Kervella, M. Génard, and S. Li. 2003. Analysis of genotypic variation of sugar and acid contents in peaches and nectarines through the principle component analysis. Euphytica 132:375-384.

Zampini, M., E. Wantling, N. Phillips, and C. Spence. 2008. Multisensory flavor perception: Assessing the influence of fruit acids and color cues on the perception of fruit-flavored beverages. Food Qual. Prefer. 19:335-343.

Zhang, H. and Y. Ge. 2016. Dynamics of sugarmetabolic enzymes and sugars accumulation during watermelon (Citrullus lanatus) fruit development. Pak. J. Bot. 48:2535-2538.

Zhu, Q., P. Gao, S. Liu, Z. Zhu, S. Amanullah, A.R. Davis, and F. Luan. 2017. Comparative transcriptome analysis of two contrasting watermelon genotypes during fruit development and ripening. BMC Genomics 18(1):3. 